\title{
La carence protidique des gastrectomisés
}

Depuis 25 ans, la gastrectomie est considérée comme Гopération de choix pour le traitement de Tulcère gastro-duodénal. La technique chirurgicale perfectionnée en fait une intervention fort peu choquante, et le malade s'adapte de façon étonnamment rapide à se passer des deux tiers de son estomac. Cependant, à mesure que le nombre des opérés s'accroît et que le recul augmente, certaines sêquelles de la gastrectomie se multiplient: Гulcère peptique jéjunal, par exemple, complication réservée autrefois à la gastro-entérostomie, n'épargne pas les estomacs réséqués. Mais c'est sur le plan nutritionnel que les inconvénients de la gastrectomie semblent parfois le plus sérieux: toute une série de documents ont été publiés en France, depuis les premieres observations de A. Lambling et M. Conte (Bull. Mém. Soc. Méd. Hop., Paris,

Editorial

237

11 févr. 1949, p. 151) et de R. Cattan et coll. (Arch, malad. appar. digest., nov./déc. 1949, p. 1168), documents qui ont été colligés en Suisse par B. Rílliet (Rev. méd. Suisse romande, juin 1951, p. 407).

A côté de ces cas graves, qui restent heureusement des exceptions, il existe un déséquilibre protidique frequent et latent, auquel L. Hart-mann, P. Guérín et jR. Fauvert (Arch, malad. appar. digest., juillet/ août 1952, p. 721) consacrent une etude fort suggestive. Ces auteurs ont analyse le syndrome biologique de 100 réséqués provenant d'une série homogène, dont les résultats, après 2 à 7 ans, se décomposaient en 63 succès complets, 28 résultats moyens (mais n'empêchant pour-tant pas l'opéré de travailler en plein), et 9 échecs, dont 4 seulement par troubles digestifs: douleur épigastrique, dumping-syndrom ou diarrhée rebelle.

Ils ont ensuite divisé $\Gamma$ ensemble de leurs malades selon leur état de nutrition: dans le premier groupe, comprenant 18 sujets ayant un poíds de $10 \mathrm{~kg}$. ou plus en-dessous du poids ideal, Texploration biologique met en evidence, dans la totalité des cas, une diminution impor-tante des protides totaux et de $\Gamma$ hémoglobine totale circulants. Le second groupe comporte 52 observations oil le poids étaít légèrement insuffisant ( 5 à $9 \mathrm{~kg}$. en-dessous du poids théorique): les troubles protidiques y sont modérés, et le plus souvent dissociés. Quant aux 30 malades dont le poids étaít égal ou supéríeur au poids ideal, les perturbations protidiques sont, le plus souvent, à peine ébauchées.

Comparant alors les chiffres: $63 \%$ d'excellents résultats cliniques, avec $20 \%$ seulement de syndrome biologique normal, ils en concluent que $40 \%$ environ des opérés présentent un état de dénutritionprotidique modérê ou díscret, qui reste latent; cela concerne surtout deux categories d'âge extremes: les malades de moins de 30 ans, et ceux de plus de 60 ans.

Les consequences thérapeutiques à tirer de ces faits sont avant tout diététiques: il faut contrôler longtemps $\Gamma$ alimentation des gastrectomisés, assurer un apport abondant de protides facilement assimilables, et compenser la digestion gastrique insuffisante. Cette carence retentit d'ailleurs sur tout l'organisme, et, récemment, G. Brouet et coll. (Paris méd. 1951, p. 64) a poussé un cri d'alarme en signalant 
la frequence de la tuberculose pulmonaire dans les suites de la resection gastrique. C'est un nouvel aspect du problème qui mérite d'etre pris en sérieuse consideration, et doit pousser le gastro-entérologiste à examiner à fond le poumon de ses ulcéreux, avant de les confier au chirurgien. M. D.

$16^{*}$ 